\title{
Diseño de guías de laboratorio para desarrollar habilidades profesionales en la asignatura Automatización del programa de ingeniería industrial ${ }^{1}$
}

\author{
Leonardo Emiro Contreras Bravo ${ }^{2}$ \& Julián Alfonso Tristancho Ortiz ${ }^{3}$, \\ Universidad Distrital Francisco José de Caldas, Colombia \\ Karolina González Guerrero ${ }^{4}$ \\ Universidad Militar Nueva Granada, Colombia
}

Recibido, junio 19 de 2015

Concepto evaluación, septiembre 14 de 2015

Aceptado, octubre 15 de 2015
Referencia: Contreras Bravo, L.; Tristancho Ortiz, J; González Guerrero, K. (2015). "Diseño de guías de laboratorio para desarrollar habilidades profesionales en la asignatura Automatización del programa de ingeniería industrial ". Revista Academia y Virtualidad, 8, (2), 112-122

\section{Resumen}

En este documento se presenta un proyecto piloto implementado con los estudiantes de automatización de Ingeniería Industrial de la Universidad Francisco José de Caldas. Primero se trabaja un marco teórico y referencial asociado con el aprendizaje activo, habilidades profesionales y metodologías de enseñanza tradicional.

En la segunda parte se realiza una propuesta de evaluación del tipo de enseñanza por una metodología que permita una mayor participación y responsabilidad de parte del alumno en su propio aprendizaje y que presente una oportunidad de desarrollo de habilidades profesionales. Finalmente se presentan algunos resultados de la elaboración y aplicación de las guías de laboratorio.

Palabras clave: formación de ingenieros, guía de laboratorio, herramientas de aprendizaje activo, prácticas de laboratorio, TIC.

1. Artículo de reflexión-investigación. Grupo de investigación "Diseño, modelamiento y simulación (DIMSI), adscrito a la Facultad de Ingeniería de la Universidad Distrital Francisco José de Caldas. Producto de la investigación "Generación y validación de contenidos didácticos para el desarrollo de habilidades de estudiantes de ingeniería por medio de la implementación de TIC”.

2. Ingeniero mecánico; Magíster en materiales y procesos de manufactura; Director grupo DIMSI; Docente de planta, Universidad Distrital José de Caldas. Correo: leonardo.contreras@udistrital.edu.co

3. Ingeniero mecánico; Magíster en Ingeniería; Doctor en Ingeniería; Docente de planta, Universidad Distrital Francisco José de Caldas. Correo: julian. tristancho@udistrital.edu.co

4. Licenciada en Electrónica; Magíster en Educación; Doctora (c) en Educación; Docente de planta, Universidad Militar Nueva Granada. Correo: karolina. gonzalez@unimilitar.edu.co 


\section{Design of lab guidelines to develop professional skills of subject Automation of industrial engineering program}

\section{Abstract}

This paper describes a pilot project implemented with an automation course of industrial engineering at Universidad Distrital Francisco José de Caldas. Firstly, we work a theoretical and a referential framework related to active learning, professional skills and traditional teaching methodologies. Secondly, a new proposal of a teaching methodology by giving a greater participation and responsibility by students who may manage their own learning and providing a chance to develop professional skills. Finally, some results of such a development and implementation of lab guidelines.

Keywords: engineering training, lab guidelines, active learning tools, lab practice, ICT.

\section{Desenho de guias de laboratório para desenvolver habilidades profissionais na matéria automatização do programa de engenharia industrial}

\section{Resumo}

Neste documento apresenta-se um projeto piloto operacionalizado com estudantes de automatização de Engenharia Industrial da Universidade Francisco José de Caldas. Primeiro trabalha-se num quadro teórico e referencial associado com a aprendizagem ativa, habilidades profissionais e metodologias de ensino tradicional. Na segunda parte realiza-se uma proposta de avaliação do tipo de ensino por uma metodologia que permita uma maior participação e responsabilidade por parte do aluno na sua própria aprendizagem e que presente uma oportunidade de desenvolvimento das habilidades profissionais. Finalmente presentam-se alguns resultados da elaboração e aplicação das guias de laboratório.

Palavras chave: formação de engenheiros, guia de laboratório, ferramentas de aprendizagem ativa, práticas de laboratório, TIC.

\section{Introducción}

La experiencia recogida como estudiantes de ingeniería en el pasado y también como docentes en la actualidad; asimismo, la interacción con otros estudiantes a niveles en los que se cuestiona no solamente los conocimientos trasmitidos, sino que se indaga la habilidad para poner en situación real lo aprendido, buscando siempre el "saber hacer", y no sólo el "saber", nos ha permitido visualizar que como ingenieros debemos poseer diversas habilidades y competencias profesionales para adaptarse exitosamente a los cambios que están ocurriendo en el mundo (globalización, avance tecnológico y nuevas tecnologías), tales como: funcionar en equipos multidisciplinarios, comprender los posibles impactos de las soluciones de ingeniería y compromiso con el aprendizaje durante toda la vida, entre otras.

Pero estas habilidades parecen no encontrarse satisfechas deltodo frentealasmetodologíastradicionales deenseñanza de cursos de ingeniería. Surge entonces la pregunta de si las metodologías actuales para abordar el proceso enseñanzaaprendizaje desarrollan estas características o existen otras más adecuadas. Esta duda se empieza a despejar a partir de trabajos como el desarrollado en el programa de Ingeniería Mecánica del M.I.T (Suh, 2003), en el cual se 
Diseño de guías de laboratorio para desarrollar habilidades profesionales en la asignatura Automatización del programa de ingeniería industrial

demuestra que los estudiantes universitarios ingresan con un alto nivel de confianza en sí mismos y en el desarrollo de habilidades profesionales en el campo universitario, pero cuando salen esos niveles son notoriamente bajos. Shuman (2005) también demostró en su trabajo sobre habilidades profesionales que el desarrollo de habilidades profesionales no es muy fuerte en el sistema educativo universitario.

La práctica académica de la universidades se ha concentrado en una clase magistral caracterizada por una fuerte transmisión de conocimientos, monótona y de pocos recursos tecnológicos que ayuden al estudiante a entender y comprender los diferentes fenómenos (Van Dijk, 1999), cuando en realidad también se pueden proporcionar herramientas para que los estudiantes construyan su propio aprendizaje. Incluso algunos autores han hecho referencia a que los estudiantes prestan muy poca atención a la clase, aproximadamente $50 \%$ piensan en cosas diferentes a la clase, y cerca de $15 \%$ del tiempo fantasean (Millton, 1986).

Otros son un poco más fuertes en sus investigaciones expresando que el estudiante sólo mantiene la concentración en la misma por 15 minutos y que recuerdan los conceptos entregados por el docente sólo en los 15 minutos iniciales y finales de clase aproximadamente (Prince, 2004).

Para solucionar este tipo de impases en los últimos años se han implementado metodologías de enseñanzaaprendizaje (aprendizaje activo, aprendizaje significativo, modelos autoestructurantes y aprendizaje cooperativo) que parten de la hipótesis que el estudiante es el verdadero responsable de su aprendizaje y es el único que lo puede construir.

Éstas podrían contribuir de una manera más eficaz a conseguir las cualidades que la sociedad requiere de nuestros profesionales y a las que puede colaborar el docente con apoyo de tecnologías y con un cambio en el enfoque pedagógico (Gil 1997) (Esquembre 2004).

\section{Generalidades}

Los cursos del área de diseño y manufactura a la que pertenece la asignatura automatización tienen un fuerte componente teórico, solución de problemas tipo y algunos laboratorios. El nivel de dificultad que presentan estos cursos debido a su intensidad genera muchos problemas de apropiación del conocimiento por parte de los estudiantes. De ahí que se haya planteado un cambio metodológico en la forma como se dictan las clases y sobre todo en el nivel de participación de los discentes para alcanzar un aprendizaje activo, mejorando el nivel de retención.

\section{Identificación de habilidades}

La función de un profesional en ingeniería siempre está ligada a la manipulación de materiales, energía e información. La formación de ingenieros en la universidad debe ser dinámica siguiendo los cambios tecnológicos, las necesidades sociales e industriales actuales. Varios autores han tratado de establecer cuál es el perfil, en cuanto a sus habilidades, que un ingeniero debe tener al momento de terminar su formación universitaria de pregrado. Por ejemplo, la organización $\mathrm{ABET}^{5}$ define las habilidades que un ingeniero debe tener al momento de terminar su formación, tal como aparecen en la tabla 1.

\begin{tabular}{|c|c|}
\hline Habilidades cognitivas & Habilidades profesionales \\
\hline Aplicar conocimientos & $\begin{array}{c}\text { Funcionar en equipos } \\
\text { multidisciplinarios }\end{array}$ \\
\hline $\begin{array}{c}\text { Diseñar y realizar experimentos; } \\
\text { analizar e interpretar datos }\end{array}$ & Responsabilidad ética y profesional \\
\hline $\begin{array}{c}\text { Diseñar un sistema, componente } \\
\text { o proceso para satisfacer } \\
\text { necesidades teniendo en cuenta } \\
\text { sus impactos }\end{array}$ & Comunicarse efectivamente \\
\hline $\begin{array}{c}\text { Identificar, formular y solucionar } \\
\text { problemas de ingeniería }\end{array}$ & $\begin{array}{c}\text { Comprender los posibles impactos de } \\
\text { las soluciones de ingeniería }\end{array}$ \\
\hline $\begin{array}{c}\text { Usar técnicas, habilidades e } \\
\text { instrumentos modernos de } \\
\text { ingeniería }\end{array}$ & $\begin{array}{c}\text { Compromiso con el aprendizaje } \\
\text { durante toda la vida }\end{array}$ \\
\hline
\end{tabular}

Tabla 1. Habilidades esperadas de formación para un ingeniero Fuente: adaptación de Shuman et al, 2005.

5. ABET (acrónimo de Accreditation Board for Engineering and Technology). Entidad no gubernamental, sin ánimo de lucro, dedicada a la acreditación universitaria de ciencias aplicadas, ingeniería y tecnología. Ha acreditado 3100 programas de 660 instituciones en 23 países. 
Al analizar las habilidades necesarias por un ingeniero recién graduado, según lo muestra la tabla 1, se puede ver que las técnicas tradicionales de enseñanza no pueden llegar a fomentar muchas de las experiencias necesarias en su desarrollo. Las técnicas tradicionales están centradas en la labor del docente (presentaciones magistrales, solución de problemas teóricos planteados por el docente, prácticas de laboratorio completamente definidas en cuanto a metodologías y resultados, etc.), dejando un papel completamente pasivo a los discentes. Según Chrobak (1996), la educación se divide claramente en dos tipos fundamentales: la enseñanza pasiva y la activa, refiriéndose al rol que el estudiante cumple durante su propia formación. La enseñanza pasiva logra tener niveles de retención inferiores a 50\%, mientras que la enseñanza activa obtiene niveles superiores a 70\%. Estos resultados han sido demostrados por varios autores como Hadim et al (2000), Caro \& Reyes (2003), Ruiz et al (2006) y Fernández-Sánchez \& Millán (2013).

Algunos autores asimismo han mostrado relación alguna con el hecho de hablar de habilidades profesionales con competencias específicas, partiendo del hecho que las competencias pueden clasificarse en genéricas o transversales, específicas y básicas (Ramírez, Salas \& Bahamón, 2011). Así las cosas, expresan que las específicas son propias de cada profesión y le dan identidad a una ocupación (Tobón, 2006), pero se pueden subdividir en cognitivas que preparan al estudiante para saber y conocer con metodologías apropiadas para ello (Caicedo, 2014); las comunicativas permiten trascender, participar y transmitir conocimientos, hacerse entender, compartir y convencer; y las investigativas forman para continuar un proceso de aprendizaje constante (Ramírez, Salas \& Bahamón, 2011); las investigativas y las laborales están asociadas a la productividad y la competitividad (Fajardo, Fonseca \& Bahamón, 2015).

\section{Aprendizaje activo en la formación de ingenieros}

Kolb \& Fry (1975) proponen que para aprender algo se debe trabajar o procesar la información recibida a partir de una experiencia directa y concreta o bien de una experiencia abstracta (por ejemplo, una lectura). Estas experiencias se convierten en conocimiento cuando se elaboran de manera reflexiva o experimentan de forma activa. El aprendizaje óptimo ocurre cuando se trabajan de manera consecutiva cada una de las fases descritas anteriormente. En la figura 1 se puede observar el modelo Kolb de aprendizaje.

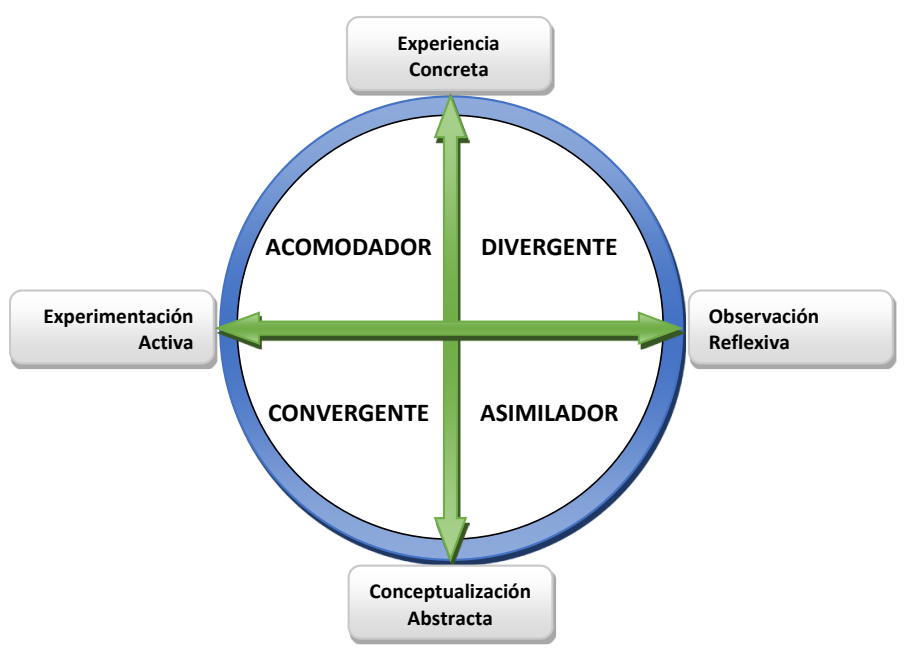

Figura 1. Modelo de aprendizaje de Kolb Fuente: adaptación a partir de Sharp, 1997.

En la práctica, la mayoría de las personas se especializan en una de las fases o máximo en dos, por lo cual se pueden describir cuatro tipos de estudiantes: activo, reflexivo, teórico y pragmático. Esto supone que se debe tener un método de aprendizaje que cubra las cuatro fases descritas por Kolb, a fin de garantizar que los estudiantes logren apropiarse el conocimiento de manera adecuada.

El objetivo de la educación en ingeniería se podría resumir en preparar a los estudiantes para la práctica de la ingeniería en particular para el uso de los tres recursos fundamentales: energía, materiales e información. La forma de hacer que los estudiantes puedan superar las cuatro etapas descritas por Kolb durante su proceso educativo es el uso de laboratorios de ingeniería. Según Feisel \& Rosa (2005), los laboratorios en ingeniería se pueden clasificar en tres tipos básicos:

- Desarrollo: para resolver cuestiones específicas acerca de la naturaleza, las cuales deben ser 
Diseño de guías de laboratorio para desarrollar habilidades profesionales en la asignatura Automatización del programa de ingeniería industrial

contestadas antes que pueda continuar un proceso de diseño o desarrollo. Pensado para resolver una pregunta específica de importancia inmediata.

- Investigación: para explorar el borde del conocimiento que puede ser generalizado y esquematizado, pudiendo no tener uso específico en la actualidad.

- Docencia: para validar el comportamiento de los modelos matemáticos planteados, contrastándolos con el comportamiento real de los objetos. También pueden ser del tipo de capacitación tecnológica sobre el uso de herramientas computacionales o físicas.

El tipo de laboratorio más usado en los procesos formativos de pregrado en ingeniería es el de docencia. En ellos el estudiante casi nunca se enfrenta a desarrollar una metodología que le permita extraer los datos necesarios para evaluar un nuevo dispositivo o crear un nuevo componente (Feisel \& Rosa, 2005). Por tanto, la inclusión de medios y tecnologías, especialmente en la Educación Superior, representa retos y cambios en las estrategias de enseñanza, en cuanto al quehacer pedagógico que asume el docente (De Miguel, 2006).

De otra parte, la integración de las prácticas o laboratorios en el proceso de diseño o evaluación permitiría integrar de manera más efectiva las cuatro etapas que propone el modelo de Kolb. Por ello, Feisel \& Rosa (2005) plantean que los objetivos que todo laboratorio debe cumplir para lograr su cometido formativo son las expuestas en la tabla 2.

Muchas de las prácticas clásicas de laboratorio no pueden cumplir con los objetivos propuestos en la tabla 2, debido a dificultades técnicas (diseño de equipos) o metodológicas (diseño de la práctica). Lo que sí queda claro de lo mostrado en la tabla 2 es que una práctica diseñada para cumplir con los objetivos propuestos por Feisel \& Rosa (2005), impactaría en gran medida en el proceso de enseñanza permitiendo que el ciclo de Kolb se implemente con mayor facilidad.

Los recursos tecnológicos en un laboratorio de automatización pueden integrarse con metodología

\begin{tabular}{|c|c|c|}
\hline ID & Área & Objetivo \\
\hline 1 & Instrumentación & $\begin{array}{l}\text { Motivar la correcta selección de sensores, } \\
\text { instrumentación y/o software para hacer la } \\
\text { medición de las variables físicas. }\end{array}$ \\
\hline 2 & Modelos & $\begin{array}{l}\text { Identificar claramente las ventajas y limitaciones } \\
\text { que poseen los modelos teóricos usados, con } \\
\text { respecto a los resultados del mundo real. }\end{array}$ \\
\hline 3 & Experimento & $\begin{array}{l}\text { Diseñar el procedimiento experimental que } \\
\text { se debe seguir para obtener los resultados o } \\
\text { mediciones deseadas. }\end{array}$ \\
\hline 4 & Análisis de datos & $\begin{array}{l}\text { Diseñar el método de extracción adecuada de la } \\
\text { información necesaria desde los datos capturados, } \\
\text { tener la capacidad de interpretar los resultados, } \\
\text { identificando los problemas y sacar conclusiones } \\
\text { acordes a un nivel científico adecuado. }\end{array}$ \\
\hline 5 & Diseño & $\begin{array}{l}\text { Tener la capacidad de crear procedimientos, } \\
\text { metodologías o nuevos dispositivos que permitan } \\
\text { obtener mediciones necesarias. }\end{array}$ \\
\hline 6 & $\begin{array}{l}\text { Realimentación } \\
\text { positiva }\end{array}$ & $\begin{array}{l}\text { Tener la capacidad de aprender de los errores } \\
\text { cometidos durante la prueba, debido a equipos, } \\
\text { metodología, construcción, etc., permitiendo } \\
\text { usar esos errores para plantear soluciones más } \\
\text { adecuadas o mejoras de su experimento. }\end{array}$ \\
\hline 7 & Creatividad & $\begin{array}{l}\text { Usar pensamiento creativo para la solución de } \\
\text { problemas a partir de las herramientas disponibles } \\
\text { y extrapolar a un problema del mundo real. }\end{array}$ \\
\hline 8 & Psicomotor & $\begin{array}{l}\text { Demostrar competencia en el uso y selección de } \\
\text { diferentes herramientas y recursos de ciencias } \\
\text { aplicadas e ingeniería. }\end{array}$ \\
\hline 9 & Seguridad & $\begin{array}{l}\text { Identificar claramente los riesgos y problemas } \\
\text { ambientales que se podrían tener durante la } \\
\text { práctica, mitigando sus posibles efectos. }\end{array}$ \\
\hline 10 & Comunicación & $\begin{array}{l}\text { Tener la capacidad de comunicar efectivamente el } \\
\text { método usado para la práctica, con sus resultados } \\
\text { y conclusiones. }\end{array}$ \\
\hline 11 & Trabajo en equipo & $\begin{array}{l}\text { Trabajar efectivamente en equipos, incluyendo } \\
\text { estructuración individual y responsabilidad } \\
\text { conjunta, junto con la asignación de roles y } \\
\text { responsabilidades. }\end{array}$ \\
\hline 12 & $\begin{array}{l}\text { Ética en el } \\
\text { laboratorio }\end{array}$ & $\begin{array}{l}\text { Comportamiento ético en la presentación de } \\
\text { resultados, incluyendo el reporte de información } \\
\text { objetiva. }\end{array}$ \\
\hline 13 & $\begin{array}{l}\text { Conciencia } \\
\text { sensorial }\end{array}$ & $\begin{array}{l}\text { Desarrollar el sentido común originario } \\
\text { para realizar juicios de ingeniería sobre } \\
\text { comportamientos y problemas }\end{array}$ \\
\hline
\end{tabular}

Tabla 2. Objetivos por desarrollar en una práctica o laboratorio de docencia. Fuente: adaptación a partir de Feisel \& Rosa, 2005. 
tradicional con el fin de formar profesionales motivados por el aprendizaje permanente y el trabajo colaborativo, de tal manera que le permitan desarrollar competencias profesionales mediante la integración de los contenidos temáticos de las asignaturas y la realidad de los fenómenos, atendiendo a que la educación se debe basar en el desarrollo de competencias actitudinales y aptitudinales, ya que la adquisición de conocimiento por contenidos en sí, puede quedar revaluada en poco tiempo (Tobón, 2006).

\section{Metodología}

\section{Participantes en la asignatura Automatización}

La asignatura Automatización es de tipo electiva de la carrera Ingeniería Industrial de la Universidad Distrital Francisco José de Caldas, se encuentra en el pensum en quinto semestre. Aunque pertenece a dicha carrera, puede ser tomada por estudiantes de diferentes ingenierías de la Facultad, como Eléctrica, Electrónica y Sistemas. Ésta se ofrece a estudiantes desde 2011, y ha generado una serie de cambios en su contenido partiendo de una base única y exclusivamente neumática e hidráulica, hasta llegar a lo que tenemos hoy en día que se ha integrado los métodos de control discreto (neumática, electroneumática y PLC), con los métodos de control continuo (dos estados, PID y SCADA). Cada semestre posee dos grupos, que tienen en promedio 25 estudiantes de ambos sexos, con una intensidad horaria de dos horas semanales para clases magistrales de los temas de desarrollo teórico y dos horas prácticas de laboratorio.

\section{Introducción a la asignatura}

El curso de "automatización" se compone de tres temas principales. El primero corresponde a las bases teóricas de los procesos de automatización; en el segundo se ve la parte operativa y diseño de sistemas neumáticos e hidráulicos usados en automatización de procesos secuenciales, mientras en el tercero se cubre la teoría relacionada al control de procesos continuos.

En este curso se hace una introducción en los niveles básicos de la pirámide de la automatización (figura 2).
Mediante conceptos teóricos y prácticas desarrolladas durante el curso el estudiante podrá identificar los componentes y procesos de diseño básicos usados en los niveles de campo (sensores y actuadores) y de control (control de máquina, de procesos discretos y continuos).

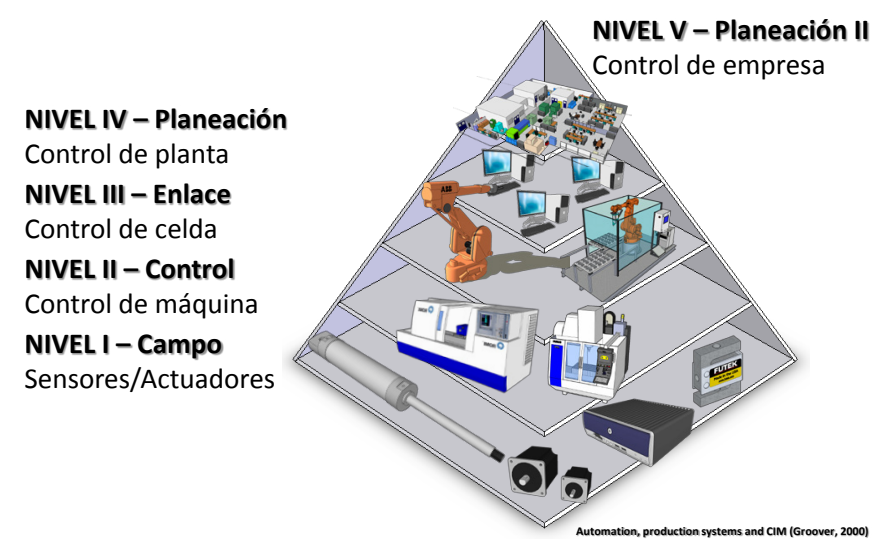

Figura 2. Pirámide de automatización

Fuente: adaptación a partir de Groover, 2000.

El objetivo principal del curso es la capacitación del estudiante de ingeniería industrial en el dominio de un lenguaje común y conocimientos básicos, necesarios para plantear, diseñar y desarrollar proyectos de automatización industrial en un grupo interdisciplinario de especialistas en el área de automatización. Las prácticas de laboratorio son fundamentales pues enfrenta al estudiante a la solución de un problema sencillo de automatización, permitiendo que aplique los conocimientos teóricos adquiridos en la parte teórica del curso.

\section{Introducción al trabajo de laboratorio}

El curso de Automatización se dicta en dos áreas: aula de clase y laboratorio de neumática e hidráulica. En el aula de clase se realiza una exposición sobre la teoría asociada, más ejemplos y ejercicios de clase.

En el laboratorio en cambio se plantea un problema de automatización el cual se soluciona en grupos y se somete a una sustentación por medio de preguntas por parte del docente y un informe de laboratorio, demostrando hallazgos y respondiendo los interrogantes planteados en las guías. El curso se considera teórico-práctico debido a su distribución de cantidad de horas (dos horas teóricas 
Diseño de guías de laboratorio para desarrollar habilidades profesionales en la asignatura Automatización del programa de ingeniería industrial

y dos horas de práctica por semana) y evaluación donde $45 \%$ de la nota final corresponde a laboratorios y proyecto final del curso.

\section{Estrategias para la mejora de la enseñanza-aprendizaje}

La metodología en general está diseñada de forma que el estudiante sea reflexivo sobre el porqué de las actividades que realiza y tenga mayor conocimiento del proceso, del resultado y de la futura aplicación industrial de lo aprendido en la práctica de laboratorio. Con ella se establece una enseñanza activa, autónoma y colaborativa aprovechando los recursos tecnológicos y con contenidos más atractivos para el estudiante.

Para las prácticas asociadas con control discreto se utilizan los bancos de neumática FESTO TP101 (neumática básica) y TP201 (electroneumática básica). En la figura 3a se muestran las partes principales de los bancos que se van a utilizar durante el desarrollo de las prácticas. Los equipos mencionados son apropiados para adquirir conocimientos básicos en materia de técnicas de control de sistemas neumáticos. Se adquieren conocimientos físicos básicos de neumática, electricidad y electroneumática; además sobre el funcionamiento, el diseño y la utilización de equipos neumáticos. Adicionalmente, se posee un equipo de automatización de procesos continuos - Festo MPS PA (figura 3b) que consta de diferentes circuitos de regulación y en el cual las funciones de control de las estaciones imitan procesos reales: mezclar, regular temperaturas, filtrar y dosificar, lo que permite estudiar y solucionar problemas del sector industrial con criterios ingenieriles a los aplicados en campo.

El sistema de estudio MPS PA cubre temas de enseñanza válidos en los sectores industriales más diversos como, por ejemplo, abastecimiento de agua, sistemas de desagüe, industria alimenticia, industria de manipulación de productos a granel, industria química y petroquímica, industria biológica y farmacéutica e industria del papel

\section{Procedimiento típico de solución de una guía}

La solución de un problema típico de automatización secuencial presenta las siguientes etapas básicas (tabla 4).

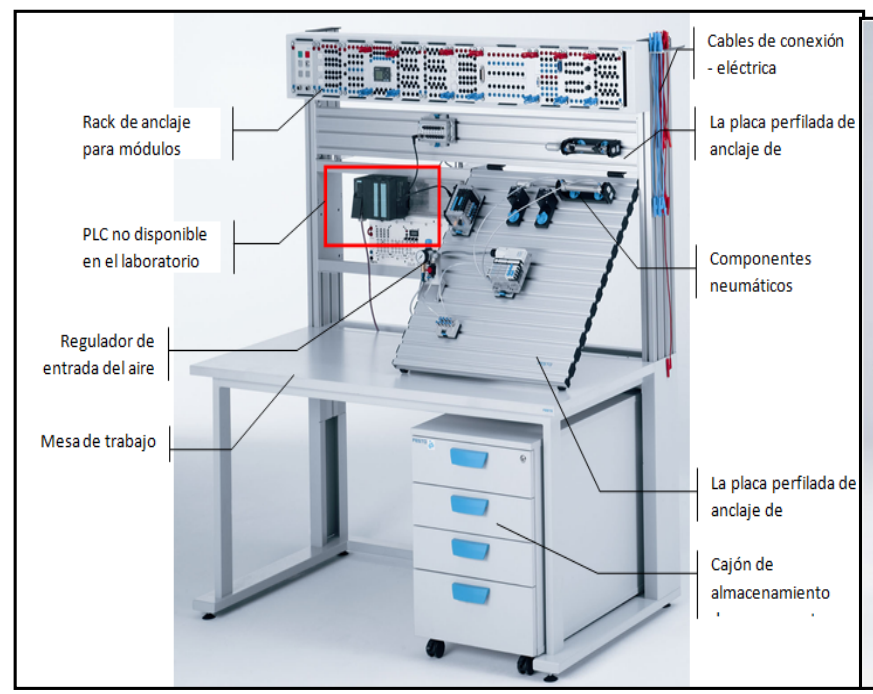

(a)

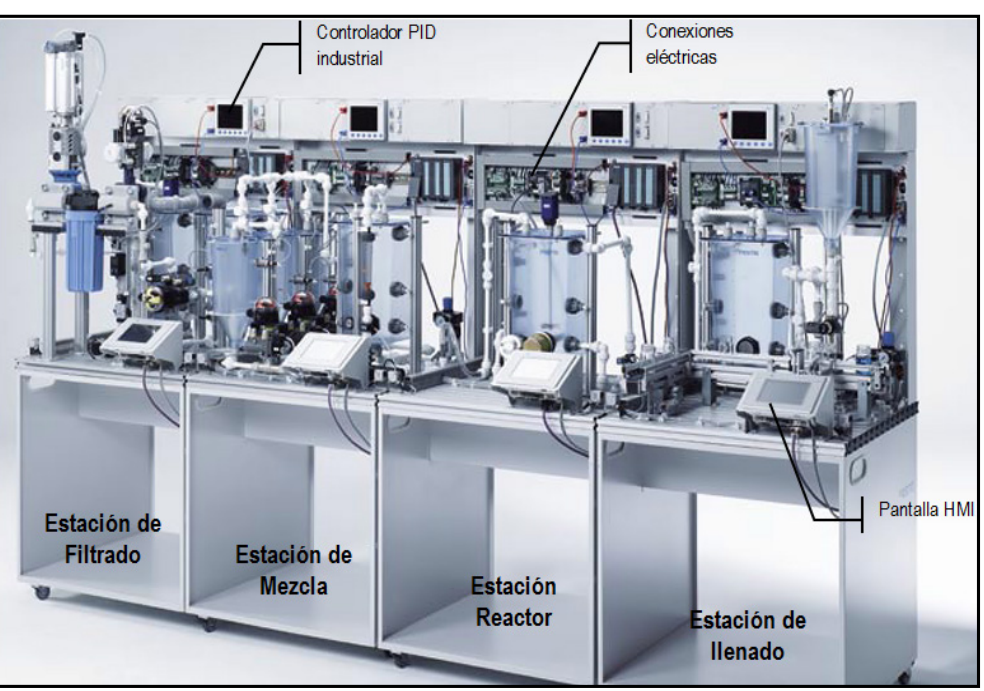

(b)

Figura 3. (a) Banco de neumática.

Fuente: www.festo.com) (b) Sistema FESTO MPS-PA 
Leonardo Emiro Contreras Bravo, Julián Alfonso Tristancho Ortiz, Karolina González Guerrero

\begin{tabular}{|c|c|}
\hline Etapa & Descripción \\
\hline $\begin{array}{l}\text { Entendimiento del } \\
\text { proceso o problema }\end{array}$ & $\begin{array}{l}\text { Analizar todo el planteamiento mediante } \\
\text { un plano de la situación. }\end{array}$ \\
\hline $\begin{array}{l}\text { Simplificación del } \\
\text { proceso }\end{array}$ & $\begin{array}{l}\text { Luego de analizar el proceso, buscar los } \\
\text { procesos o etapas que se pueden unir o } \\
\text { eliminar. }\end{array}$ \\
\hline $\begin{array}{l}\text { Determinación de } \\
\text { actuadores }\end{array}$ & $\begin{array}{l}\text { Verificar movimientos que debe realizar } \\
\text { el mecanismo. }\end{array}$ \\
\hline $\begin{array}{l}\text { Secuencia de } \\
\text { automatización y } \\
\text { sensores necesarios }\end{array}$ & $\begin{array}{l}\text { Con los actuadores y el proceso por } \\
\text { automatizar, se determina la secuencia } \\
\text { necesaria. Los sensores necesarios se } \\
\text { determinan de acuerdo con el número de } \\
\text { actuadores. }\end{array}$ \\
\hline $\begin{array}{l}\text { Diseño del circuito } \\
\text { neumático }\end{array}$ & $\begin{array}{l}\text { Con el método intuitivo o sistemático de } \\
\text { diseño, se procede a realizar un diseño } \\
\text { preliminar donde el objetivo es sólo } \\
\text { cumplir la secuencia. }\end{array}$ \\
\hline $\begin{array}{l}\text { Simulación del } \\
\text { circuito }\end{array}$ & $\begin{array}{l}\text { Mediante el software FluidSIM, es } \\
\text { posible realizar una simulación del } \\
\text { diseño, para verificar funcionamiento y } \\
\text { hacer correcciones, si son necesarias. }\end{array}$ \\
\hline $\begin{array}{l}\text { Dimensionamiento } \\
\text { de componentes }\end{array}$ & $\begin{array}{l}\text { Se debe estimar el tamaño y } \\
\text { dimensionamiento de los actuadores y } \\
\text { válvulas necesarias para implementar el } \\
\text { circuito diseñado, haciéndolo adecuado } \\
\text { para el proceso que se va a automatizar. }\end{array}$ \\
\hline $\begin{array}{l}\text { Implementación en } \\
\text { el banco del diseño } \\
\text { secuencial }\end{array}$ & $\begin{array}{l}\text { Con un grupo funcional independiente } \\
\text { buscar componentes y montarlos en el } \\
\text { banco de neumática. Un grupo funcional } \\
\text { puede ser, por ejemplo, un actuador } \\
\text { conectado a su válvula de potencia. }\end{array}$ \\
\hline $\begin{array}{l}\text { Prueba final del } \\
\text { diseño secuencial }\end{array}$ & $\begin{array}{l}\text { Conectando cada uno de los grupos } \\
\text { funcionales se realiza la prueba final de } \\
\text { funcionamiento del circuito. }\end{array}$ \\
\hline $\begin{array}{l}\text { Adición de } \\
\text { funcionalidades } \\
\text { adicionales del } \\
\text { circuito }\end{array}$ & $\begin{array}{l}\text { Una vez garantizado el funcionamiento } \\
\text { del circuito diseñado en su etapa } \\
\text { secuencial, se procede a agregar todas } \\
\text { las funcionalidades adicionales, tales } \\
\text { como temporización, señales de parada } \\
\text { o arranque, secuenciadores por presión, } \\
\text { alarmas, sistemas de visualización de } \\
\text { estado, etc. }\end{array}$ \\
\hline
\end{tabular}

Tabla 4. Secuencia de solución de la guía de laboratorio

\section{Prácticas de laboratorio}

En el desarrollo de las prácticas de laboratorio pueden existir varias sesiones para un mismo tema, dependiendo del desarrollo del temario del curso, avance por parte de los estudiantes y condiciones de disponibilidad del laboratorio. El docente informa en la clase de teoría anterior, la práctica de laboratorio que se realizará, con el objetivo que ellos puedan realizar una preparación previa. Para los laboratorios que no se realizan, el docente recomienda que los resuelvan como método de estudio para el parcial. Los estudiantes tienen a disponibilidad desde el primer día de clase el compendio de guías de laboratorios disponibles para el desarrollo de curso. Cada guía de laboratorio práctica tiene un encabezado común como el mostrado en la figura 4.

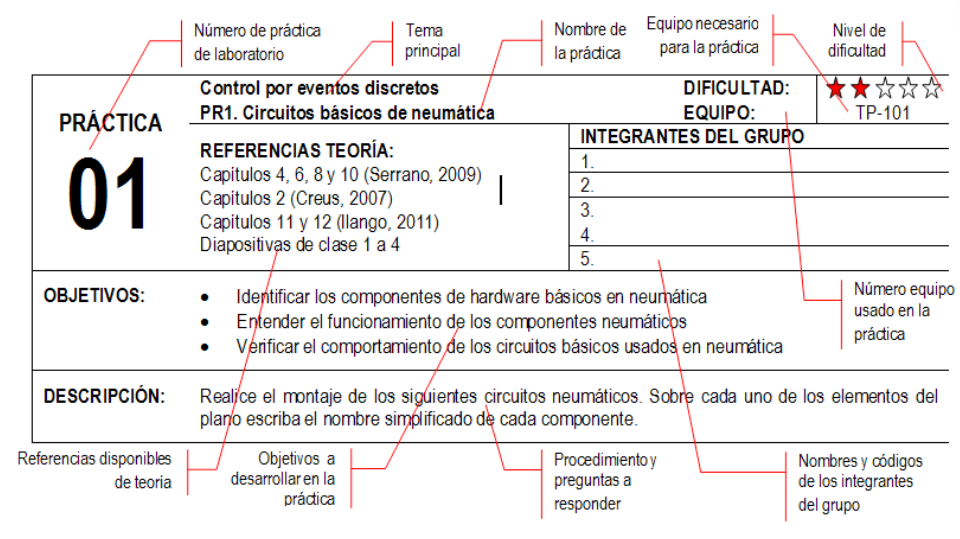

Figura 4. Partes principales de trabajos prácticos de laboratorio.

Las guías de laboratorio desarrolladas para la asignatura con el total poseen aspectos comunes (figura 5), como título de la práctica, objetivos, materiales, pasos para realizarla, bibliografía y preguntas para una mayor comprensión del proceso automatizado. Este tipo de estructura permite el trabajo colaborativo en las horas del laboratorio al estudiante (HTC) y un trabajo autónomo para poder desarrollarla fuera del curso (horas de trabajo autónomo-HTA), en la que tendrá que buscar una serie de bibliografía, algunas de las cuales es sugerida en la práctica, pero debe ser ampliada para la elaboración de un buen informe académico. De esta manera, se ayuda al desarrollo de las habilidades y/o competencias mencionadas. 
Diseño de guías de laboratorio para desarrollar habilidades profesionales en la asignatura Automatización del programa de ingeniería industrial
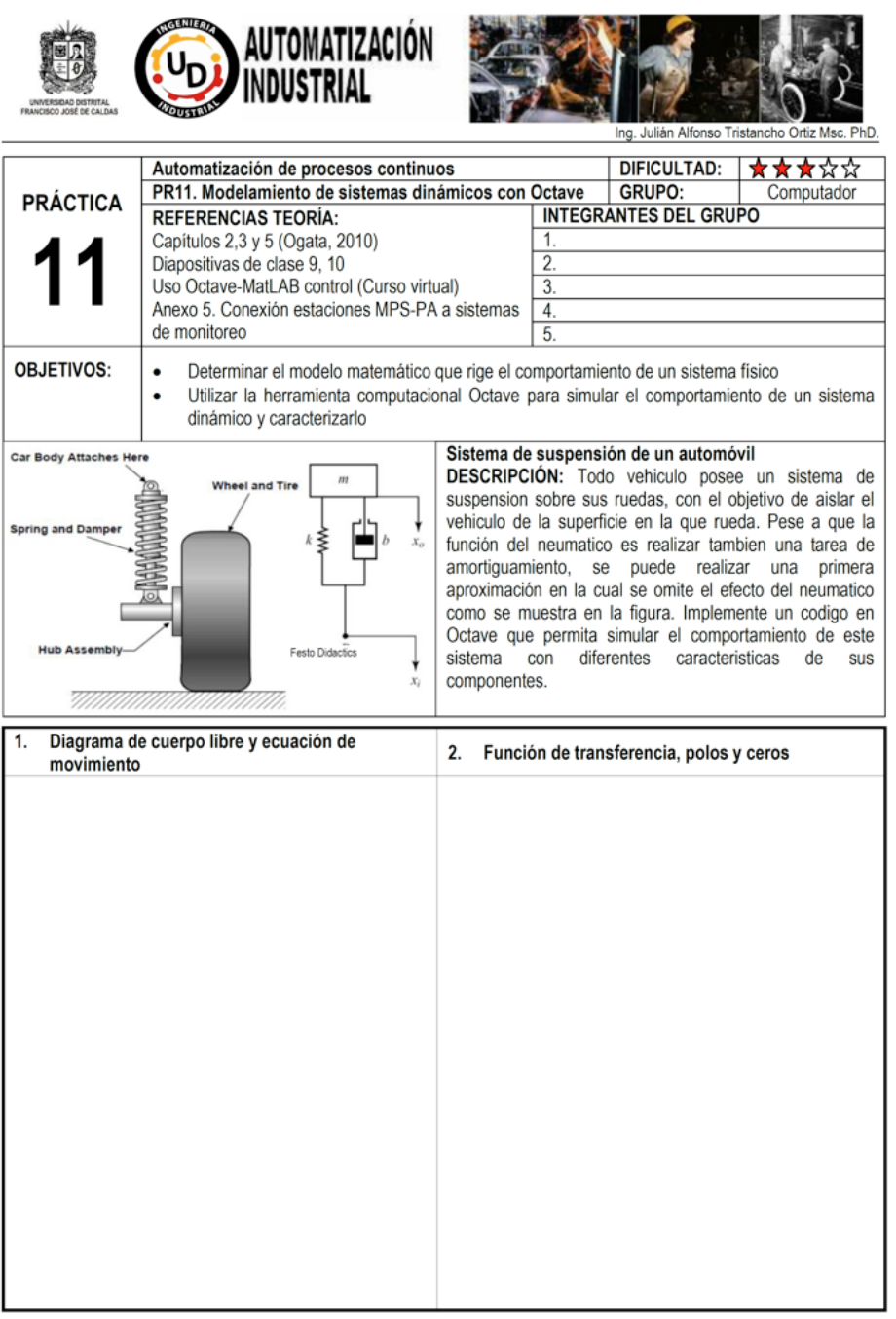

Figura 5. Ejemplo de guía completa de laboratorio.

\section{Resultados}

Las guías de laboratorio fueron diseñadas incorporando una información de carácter teórico para consultar, de esta manera los estudiantes se vuelven activos y propenden por el desarrollo de su aprendizaje, así obtienen indicadores del nivel de dificultad, lo que les permite realizar con el suficiente tiempo la preparación del laboratorio llegando a soluciones planteadas y simuladas en el software FluidSIM. Esta optimización del tiempo de estudio fuera de clase ha permitido aumentar el número de prácticas que se desarrollan en el curso, pasando de una media de 6 , antes de la implementación de las guías de laboratorio (Antes de 2013-3), a una media de 9, lo que representa un incremento de 33\% (figura 6). Éste es un aspecto

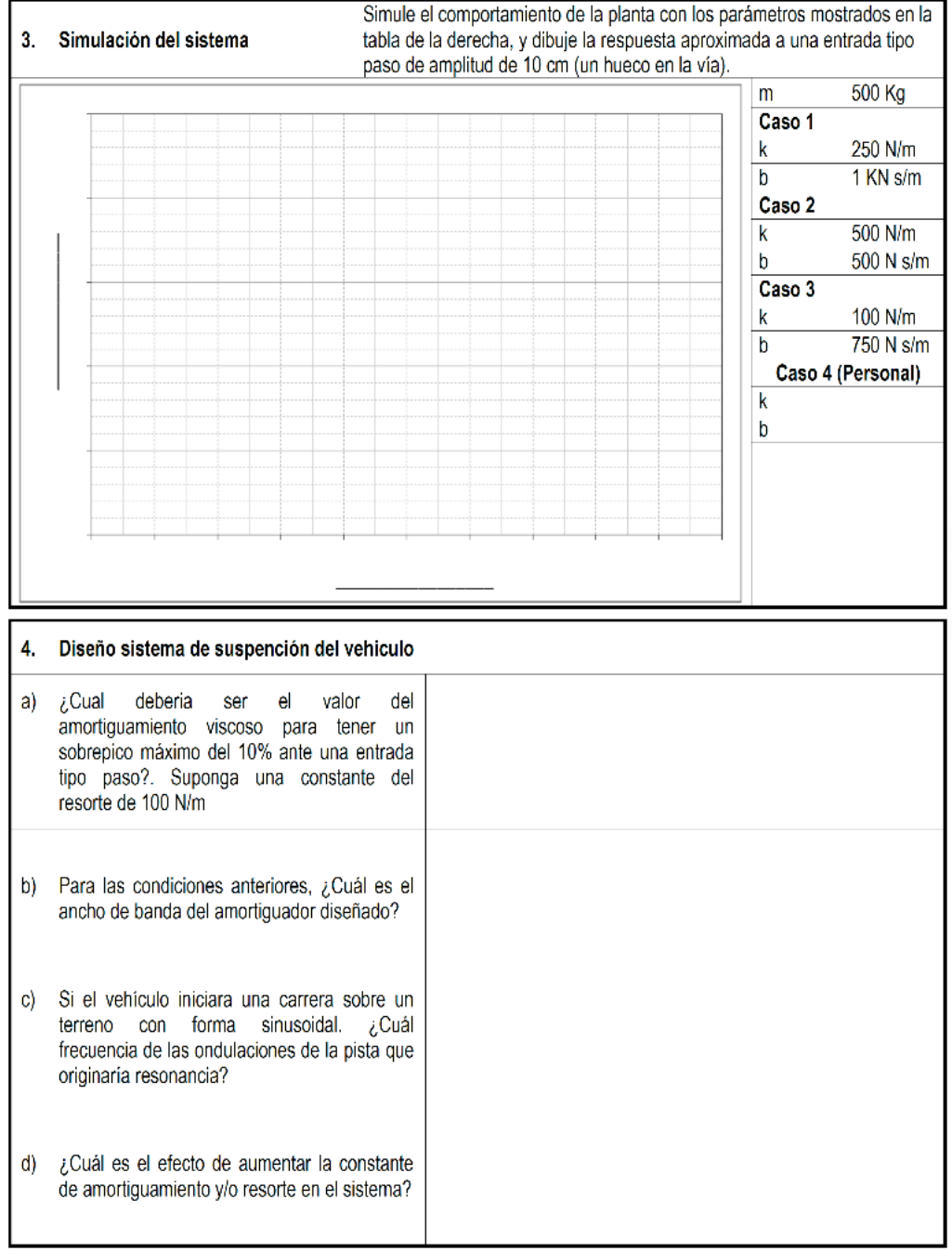

positivo debido a que en la asignatura en años anteriores se limita al manejo de elementos neumáticos en los que los estudiantes armaban diferentes circuitos que poseían los manuales de los equipos neumáticos de marca FESTO, pero no solucionaban casos o problemas reales de la industria ni realizaban propuestas de mejoras de un proceso industrial, tal como lo realizan en estos momentos al aplicar las guías.

En cuanto al rendimiento académico se puede observar en la figura 7 que la media de la nota final del curso era aproximadamente 3.3 antes de la implementación de las nuevas guías de laboratorio. Posterior a la implementación, se logra subir esa media a un valor cercano a 3.6, lo que implica un incremento del orden de $10 \%$. Cabe 
Número de prácticas realizadas por semestre

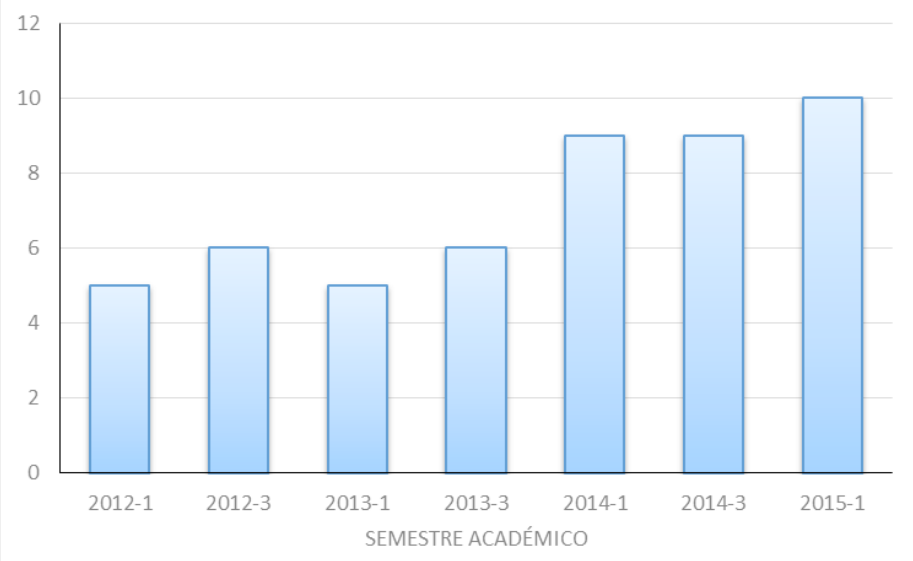

Figura 6. Histórico del número de prácticas realizadas por semestre.

mencionar que aunque pareciera poco, esta media incluye a estudiantes desertores, pues el retiro voluntario del curso antes de terminarlo es un indicador del interés de los estudiantes por éste. Ésta, por ser una materia de carácter electivo, en el pensum de ingeniería industrial, permite que los estudiantes deserten y posteriormente cancelen la asignatura sin afectar su promedio académico.

En cuanto al número de estudiantes que pierden el curso se logra pasar de una media de $30 \%$ a $18 \%$, lo que implica una disminución de $12 \%$. De esta manera se está mejorando el proceso de enseñanza-aprendizaje de la asignatura al ver que la metodología usada produce buenos resultados, y que puede continuar incorporando otras metodologías para mejorar el rendimiento académico a partir del apoyo de recursos existentes en la web, la metodología B-Learning, entre otros.

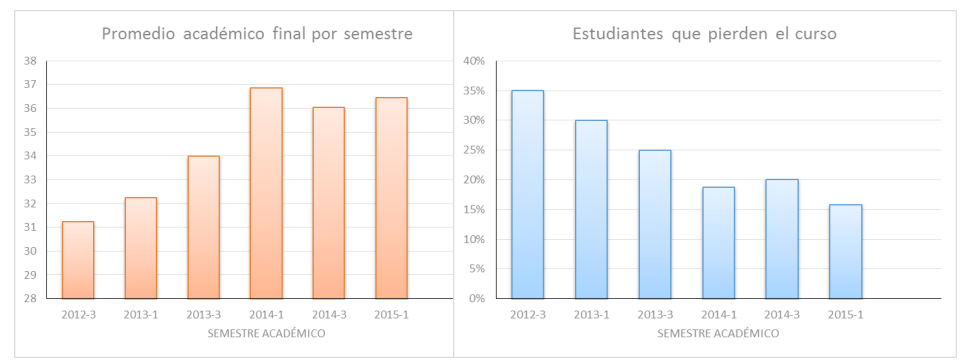

Figura 7. Histórico de rendimiento académico: Promedio nota final del curso (izquierda). Porcentaje de estudiantes que pierden el curso (derecha).

\section{Conclusiones}

El hecho de introducir nuevas metodologías de enseñanza al aula no conlleva a la desaparición de la metodología tradicional; por el contrario, es un complemento en el que el docente debe visualizar cuál de las técnicas, las herramientas educacionales o los recursos web son los más adecuados para el mejoramiento del proceso enseñanzaaprendizaje de sus educandos.

El uso de las guías de laboratorio bajo ciertas características como metodología ayuda al desarrollo de diferente tipos de competencias y habilidades en el estudiante, especialmente a ingenieros industriales que toman la asignatura, por no ser específicamente de su campo de acción ya que les amplía el panorama de su campo, les acorta la brecha entre el mundo académico y el real debido a que los equipos de laboratorio permiten automatización de procesos industriales reales a escala reducida.

La primera consecuencia directa de la implementación de guías de laboratorio con gran componente de trabajo autónomo, es la posibilidad de realización de un número mayor de prácticas. Ese incremento permite que los estudiantes se apropien de una mejor manera del conocimiento teórico desarrollado al realizar un aprendizaje del tipo activo. Como índices de valoración de resultados se tomó el promedio académico y el porcentaje de estudiantes que pierden, lográndose mejoras significativas.

\section{Referencias}

Caro, S. \& Reyes, J. Prácticas docentes que promueven el aprendizaje activo en Ingeniería Civil, Revista de Ingeniería, Núm. 18, p.p.48-55, 2003.

Chrobak, R. (1996). The Globalization and the Engineering Teaching for the XXI Century. Primer Congreso Argentino de Enseñanza en la Ingeniería. Río (Córdoba). 
Diseño de guías de laboratorio para desarrollar habilidades profesionales en la asignatura Automatización del programa de ingeniería industrial

De Miguel, M. (Coord.) (2006). Metodologías de enseñanza y aprendizaje para el desarrollo de competencias. Orientaciones para el profesorado universitario ante el espacio europeo de Educación Superior. Madrid: Alianza Editorial.

Esquembre, F; Martin, E; Cristian, W; Belloni, M. (2004) Fislets Enseñanza de la Física con Material Interactivo, Madrid, Pearson-Prentice Hall.

Fajardo J, Casallas M \& Bahamón, A. “Diseño de las guías de laboratorio para desarrollar competencias laborales especificas en el programa de ingeniería industrial de la umng”. Revista ingeniería. Vol. 10, $\mathrm{N}^{\circ} .19$, pp. 151-159. 2015

Feisel L. \& Rosa A., "The Role of the Laboratory in Undergraduate Engineering Education”, Journal of Engineering Education Volume 94, Issue 1, p.p. 121-130, January 2005

Gil, D. Martínez Torregrosa, J. (1984) Problem solving in Phisyc: A critical analisys,

Hadim H., Donskoy D., Sheppard K., Gallois B. \& Nazalewicz J., "Teaching Mechanics to Freshmen by Linking the Lecture Course to a Design Course", ASEE Conference Proceedings Search, 2000

Kolb D., \& Fry R., "Toward an applied theory of experiential learning”, in C. Cooper (ed.), Theories of Group Process, London: John Wiley. 1975

Prince, M., Does active learning work? A review of the research. Journal of Engineering Education, Vol. 93, $N^{\circ} 3,2004$
Ramírez, V. A., Salas, H. A. \& Bahamón, A. "Confrontación del modelo de calidad integral para un programa de pregrado de ingeniería industrial con las competencias y observatorio laboral". Revista Gestión Integral en Ingeniería Neogranadina, pp. 1-12. 2015

Research on Physic education. Paris. Edition du CNRS

Ruiz, D., Magallón, J. \& Muñoz, E. “Herramientas de aprendizaje activo en las asignaturas de ingeniería estructural", Ingeniería y Universidad, vol. 10, núm. 1, p.p.97-115, 2006

Sharp J.,Harb J.\& Terry R., "Combining Kolb learning styles and writing to learn in engineering classes", Journal of Engineering Education ,Vol. 86, Issue 2, pages 93-101, April 1997.

Shuman, L., Besterfield-Sacre, M. \& Mcgourty, J. "The ABET "Professional Skills" - Can They Be Taught? Can They Be Assessed? Journal of Engineering Education, Vol. 94, $\mathrm{N}^{\circ}$ 1, pp. 41-55, 2005.

Suh, N., "Transformation of Mechanical Engineering Education at M.I.T.", Journal of STEM Education, Vol. 4, No 3y4, pp. 5, 2003.

Tobón, S., Formación Basada en Competencias Pensamiento Complejo, Diseño Curricular y Didáctico, Ecoe ediciones Ltda., Bogotá, 2006.

Van Dijk, L. A., G. C. van der Berg \& H. van Keulen. "Using active instructional methods in lectures: a matter of skills and preferences.", Innovations in Education and Training International, Vol. 36, $\mathrm{N}^{\circ}$ 4, pp. 260-272, 1999.Educación Superior 